\title{
Upper urinary tract stone disease in patients with poor performance status: active stone removal or conservative management?
}

Shimpei Yamashita ${ }^{1 *}$, Yasuo Kohjimoto ${ }^{1}$, Yasuo Hirabayashi ${ }^{2}$, Takashi Iguchi ${ }^{1}$, Akinori lba ${ }^{1}$, Masatoshi Higuchi ${ }^{3}$, Hiroyuki Koike ${ }^{4}$, Takahito Wakamiya', Satoshi Nishizawa ${ }^{1}$ and Isao Hara ${ }^{1}$

\begin{abstract}
Background: It remains controversial as to whether active stone removal should be performed in patients with poor performance status because of their short life expectancy and perioperative risks. Our objectives were to evaluate treatment outcomes of active stone removal in patients with poor performance status and to compare life prognosis with those managed conservatively.

Methods: We retrospectively reviewed 74 patients with Eastern Cooperative Oncology Group performance status 3 or 4 treated for upper urinary tract calculi at our four hospitals between January 2009 and March 2016. Patients were classified into either surgical treatment group or conservative management group based on the presence of active stone removal. Stone-free rate and perioperative complications in surgical treatment group were reviewed. In addition, we compared overall survival and stone-specific survival between the two groups. Cox proportional hazards analysis was performed to investigate predictors of overall survival and stone-specific survival.

Results: Fifty-two patients (70.3\%) underwent active stone removal (surgical treatment group) by extracorporeal shock wave lithotripsy $(n=6)$, ureteroscopy $(n=39)$, percutaneous nephrolithotomy $(n=6)$ or nephrectomy $(n=1)$. The overall stone-free rate was $78.8 \%$ and perioperative complication was observed in nine patients $(17.3 \%)$. Conservative treatment was undergone by 22 patients (29.7\%) (conservative management group). Two-year overall survival rates in surgical treatment and conservative management groups were $88.0 \%$ and $38.4 \%$, respectively $(p<0.01)$ and two-year stone-specific survival rates in the two groups were $100.0 \%$ and $61.3 \%$, respectively $(p<0$. 01). On multivariate analysis, stone removal was not significant, but was considered a possible favorable predictor for overall survival $(p=0.07)$. Moreover, stone removal was the only independent predictor of stone-specific survival $(p<0.01)$.
\end{abstract}

Conclusions: Active stone removal for patients with poor performance status could be performed safely and effectively. Compared to conservative management, surgical stone treatment achieved longer overall survival and stone-specific survival.

Keywords: Poor performance status, Urolithiasis, Extracorporeal shock wave lithotripsy, Ureteroscopy, Percutaneous nephrolithotomy, Prognosis

\footnotetext{
*Correspondence: keito608@wakayama-med.ac.jp

'Department of Urology, Wakayama Medical University, 811-1 Kimiidera,

Wakayama City, Wakayaka 641-0012, Japan

Full list of author information is available at the end of the article
} 


\section{Background}

The rate of aging (65 years of age or older) worldwide is expected to rise from $7.6 \%$ in 2010 to $18.3 \%$ in 2060 as the population increases [1]. As the rate of aging rises, patients with poor performance status (PS) are also expected to increase worldwide. These patients have increased risk of urolithiasis because of various factors, including hypercalciuria associated with osteoporosis, urinary stasis, urinary tract infection and low fluid intake. Therefore, management of urolithiasis in patients with poor PS is emerging as a crucial issue in urology.

However, debate exists as to whether active stone removal should be performed in patients with poor PS. One of the reasons for such debate is that patients with poor PS have poor prognoses because of their comorbidities [2-4]. In addition, active stone removal for poor PS patients is introduced to deal with various problems, such as risks involved in their comorbidities, decreased immune competence, coexisting urinary tract infection and restriction on surgical positioning. Nonetheless, it is necessary to investigate whether active stone removal for patients with poor PS is beneficial.

To date, there have been few reports concerning the optimal management of urolithiasis in these patients. In this study, we evaluate treatment outcomes of active stone removal in patients with poor PS and compared life prognosis with those managed conservatively.

\section{Methods}

\section{Patients}

Between January 2009 and March 2016, 81 patients with Eastern Cooperative Oncology Group (ECOG) PS 3 or 4 were hospitalized for upper urinary tract calculi at the Wakayama Medical University Hospital, Hashimoto Municipal Hospital, Kinan Hospital and Rinku General Medical Center. Of these, seven patients who experienced spontaneous stone expulsion were excluded and 74 patients were enrolled in this study. This retrospective study was approved by the Institutional Review Board of Wakayama Medical University (approval number 1922).

Clinical information including age, gender, ECOG PS, medical history, Charlson Comorbidity Index (CCI), serum creatinine on admission, coexisting acute pyelonephritis, urinary drainage and stone characteristics were collected retrospectively from medical records. Urinary drainage was defined as placement of ureteral stent or nephrostomy tube on admission. Stone characteristics, including size, location and number, were assessed by non-contrast computed tomography (NCCT) and stone size was defined as the largest diameter of the major stone. In addition, data were collected on the management of any stones, such as active stone removal, including shock wave lithotripsy (SWL), ureteroscopy (URS) and percutaneous nephrolithotomy
(PCNL), and observation without operation. Patients were classified into two groups, surgical treatment group and conservative management group, based on occurrence of active stone removal. The treatment policy was left to the judgment of attending physicians, patients and their families.

\section{Outcomes}

We investigated the stone-free rate (SFR) and perioperative complications (Clavien-Dindo system grade II or more) in the operation group. Stone-free status was determined using NCCT within 3 months after operation and was defined as the absence of stones or residual fragments of less than $4 \mathrm{~mm}$. We also investigated stone-related event-free survival (EFS), with events defined as stone-related symptoms and interventions, and recurrence-free survival (RFS), with recurrence defined as new stone formation and/or regrowth of residual fragments on imaging studies as well as any stone-related events. In addition, we defined stone-specific survival (SSS) as a net survival measure representing urolithiasis survival in the absence of other causes of death, and compared overall survival (OS) and SSS between the surgical treatment group and the conservative management group.

\section{Statistical analyses}

Chi-square test, Fisher's exact test and Mann-Whitney U test were used for univariate analyses to compare variables between surgical treatment group and conservative management group. EFS, RFS, OS and SSS rates were calculated by the Kaplan-Meier method with the hospitalization date as the starting point. Univariate and multivariate analyses of OS and SSS were performed to compare the prognostic factors in a Cox proportional hazards analysis. Any $P$ values less than 0.05 were considered significant. All statistical analyses were performed using JMP Pro 12 (SAS Institute, USA).

\section{Results}

\section{Patient demographics}

Patient demographics and stone characteristics are summarized in Table 1 . The median age was 82 years (range: 36-98 years) and 51 (68.9\%) patients were females. ECOG PS was 3 in 19 (25.7\%) patients and 4 in 55 (74.3\%) patients. Sixty-one $(82.4 \%)$ patients presented acute pyelonephritis. The median stone size was $11.5 \mathrm{~mm}$ (range: $2-53 \mathrm{~mm}$ ). More information about patient demographics and stone characteristics can be found in Additional file 1: Table S1.

Of 74 patients included in this study, 22 patients (29.7\%) did not undergo stone removal and were classified as conservative management group. In conservative management group, 15 patients $(68.2 \%)$, three patients 
Table 1 Patient demographics

\begin{tabular}{|c|c|c|c|c|}
\hline & \multirow[t]{2}{*}{ Total } & \multicolumn{2}{|l|}{ Management } & \multirow{2}{*}{$\begin{array}{l}p \text { value } \\
\text { (surgical } \\
\text { treatment vs. } \\
\text { conservative } \\
\text { management) }\end{array}$} \\
\hline & & Surgical treatment & Conservative management & \\
\hline No. patients (\%) & 74 & $52(70.3)$ & $22(29.7)$ & \\
\hline Age, years & $82(36-98)$ & $76(36-92)$ & $86(68-98)$ & $<0.01$ \\
\hline Female, n (\%) & $51(68.9)$ & $33(63.5)$ & $18(81.8)$ & 0.17 \\
\hline ECOG PS, n (\%) & $19(25.7)$ & $16(30.8)$ & $3(13.6)$ & 0.15 \\
\hline 3 & $55(74.3)$ & $36(69.2)$ & $19(86.4)$ & \\
\hline \multicolumn{5}{|l|}{4} \\
\hline Charlson comorbidity index & $2(0-8)$ & $2(0-6)$ & $2(0-8)$ & 0.82 \\
\hline Serum creainine on admission, mg/dL & $1.01(0.33-5.49)$ & $0.89(0.44-5.49)$ & $1.26(0.33-3.83)$ & 0.28 \\
\hline History of urinary calculi, n (\%) & $20(27.0)$ & $16(30.8)$ & $4(18.2)$ & 0.39 \\
\hline Coexisting acute pyelonephritis, n (\%) & $61(82.4)$ & $40(76.9)$ & $21(95.5)$ & 0.09 \\
\hline Urinary drainage & $61(82.4)$ & $43(82.7)$ & $18(81.8)$ & 1.00 \\
\hline Bilateral stone, n (\%) & $7(9.5)$ & $7(13.5)$ & $0(0.0)$ & 0.09 \\
\hline \multicolumn{5}{|l|}{ Stone position, n (\%) } \\
\hline Kidney & $17(23.0)$ & $14(26.9)$ & $3(13.6)$ & \\
\hline Ureter & $44(59.5)$ & $28(53.9)$ & $16(72.7)$ & \\
\hline Kidney and ureter & $13(17.6)$ & $10(19.2)$ & $3(13.6)$ & \\
\hline Stone size, mm & $11.5(2-53)$ & $12(2-53)$ & $10.5(4-35)$ & 0.82 \\
\hline Multiple stones, n (\%) & $41(55.4)$ & $28(53.9)$ & $13(59.1)$ & 0.79 \\
\hline
\end{tabular}

Abbreviations: ECOG, Eastern Cooperative Oncology Group, PS performance status Continuous variables are shown in "median (range)" form

(13.6\%) and four patients (18.2\%) had a ureteral stent, a nephrostomy tube and neither of these, respectively. A further $52(70.3 \%)$ patients underwent active stone removal and were classified as surgical treatment group patients. The median interval between first admission and definitive therapy in surgical treatment group was 41 days (range: $2-243$ days). The patients with acute pyelonephritis at admission underwent surgical treatment after improvement of infections. Comparing patients in surgical treatment group and those in conservative management group, the median age of patients in conservative management group was 86 years and significantly older than in surgical treatment group $(p<0.01)$. Coexisting acute pyelonephritis and unilateral stones seemed to be more frequently observed in conservative management group compared with surgical treatment group $(p=0.09$ and $\mathrm{p}=0.09$, respectively).

\section{Treatment outcomes in surgical treatment group}

Patients in operation group were treated by SWL $(n=6$, $8.8 \%)$, URS ( $n=39,52.7 \%)$, PCNL $(n=6,8.1 \%)$ or nephrectomy $(n=1,1.4 \%)$. All PCNL cases were performed in a prone split-leg position and combined with retrograde flexible ureteroscopy. Overall SFR in operation group was $78.8 \%$ and by the operative treatment method, $50.0 \%$ in SWL, $87.2 \%$ in URS and $50.0 \%$ in PCNL (Table 2). Nine patients (17.3\%) experienced perioperative complications. Postoperative pyelonephritis (Clavien-Dindo system grade II) were observed in eight patients (one out of six SWL patients, six out of 39 URS patients and one out of six PCNL patients) and other complication, namely pseudoaneurysm (Clavien-Dindo system grade III), was observed in one out of six PCNL patients (Table 2).

Two-year and five-year RFS rates in surgical treatment group were $60.2 \%$ and $42.1 \%$, respectively (Fig. 1a). On the other hand, two-year and five-year EFS rates were $86.0 \%$ and $75.4 \%$, respectively (Fig. 1 b).

\section{Comparison of overall survival and stone-specific survival rates between surgical treatment group and conservative} management group

In entire cohort, nine patients $(12.2 \%)$ died of pyelonephritis or renal failure associated with urolithiasis and 17 patients $(23.0 \%)$ died of other causes during the observation period (median 23 months, range: 178 months). A total of 15 patients in conservative management group died and, of these patients, 12 patients (80.0\%), 1 patient (6.7\%) and 2 patients (13.3\%) had a ureteral stent, a nephrostomy tube and neither of these, 
Table 2 Stone-free rates and perioperative complications in operation group

\begin{tabular}{|c|c|c|c|c|c|}
\hline & \multirow{2}{*}{$\begin{array}{l}\text { No. } \\
\text { pts. }\end{array}$} & \multirow{2}{*}{$\begin{array}{l}\text { Stone- } \\
\text { free pts., } \\
n=(\%)\end{array}$} & \multicolumn{3}{|c|}{ Perioperative complications, $n=(\%)$} \\
\hline & & & Postoperative pyelonephritis & Others & Total \\
\hline SWL & 6 & $3(50.0)$ & $1(16.7)$ & $0(0.0)$ & $1(16.7)$ \\
\hline URS & 39 & $34(87.2)$ & $6(15.4)$ & $0(0.0)$ & $6(15.4)$ \\
\hline PCNL & 6 & $3(50.0)$ & $1(16.7)$ & $1(16.7)$ & $2(33.3)$ \\
\hline Nephrectomy & 1 & $1(100.0)$ & $0(0.0)$ & $0(0.0)$ & $0(0.0)$ \\
\hline Total & 52 & $41(78.8)$ & $8(15.4)$ & $1(1.9)$ & $9(17.3 \%)$ \\
\hline
\end{tabular}

respectively. Two-year OS rates in surgical treatment and conservative management groups were $88.0 \%$ and $38.4 \%$, respectively ( $p<0.01$, Fig. $2 \mathrm{a}$ ), while two-year SSS rates in surgical treatment and conservative management groups were 100.0 and $61.3 \%(p<0.01$, Fig. 2b).

\section{Associations between various parameters and overall survival}

Univariate and multivariate Cox proportional hazards regression models were used to investigate predictors of OS (Table 3). Among several predictors, age $(p<0.01)$, female $(p=0.02)$ and stone removal $(\mathrm{p}<0.01)$ were identified as significant predictors for OS on univariate analysis. Furthermore, CCI trended toward significance $(p=0.06)$. Of these factors, age (HR 1.08, 95\% CI 1.02$1.15)$ and CCI (HR 1.36, 95\% CI 1.10-1.68) were independent unfavorable predictors of OS on multivariate analysis. Stone removal was not significant, but was considered a possible favorable predictor of OS (HR 0.43, 95\% CI 0.16-1.09).

\section{Associations between various parameters and stone- specific survival}

Table 4 shows the results of univariate and multivariate Cox proportional hazards regression models of factors which predict SSS. Age $(\mathrm{p}<0.01)$ and stone removal $(\mathrm{p}<0.01)$ were significantly associated with SSS in univariate analysis. Female gender trended toward significance $(p=0.08)$. Of the factors, stone removal was the only independent predictor of SSS in multivariate analysis (HR 0.06, 95\% CI 0.00-0.43).

\section{Discussion}

We analyzed the treatment outcomes of active stone removal in patients with poor PS and compared life prognosis with those managed conservatively. In this study, we made three important clinical observations.

First, if patients who are suitable for active stone removal are appropriately selected, active stone removal could be performed safely, in spite of their poor PS. One of the main reasons for avoiding surgical treatment for patients with poor PS is the risk of perioperative complications, especially infectious disease. A review of the current literature on the management of urolithiasis in patients with spinal cord injury showed that the overall complication rate in patients with spinal cord injury is higher than in the general population, and the majority of these are infectious in nature are due to the associated medical comorbidities and chronic bacteriuria [5]. In our study, postoperative pyelonephritis was observed in $16.7 \%$ ( $1 / 6$ cases), $15.4 \%$ (6/39 cases) and $16.7 \%(1 / 6$ cases) of the patients who underwent SWL, URS and PCNL, respectively. This was higher than previously reported [6-9]. However, major perioperative complications (Clavien-Dindo system grade III or more) were
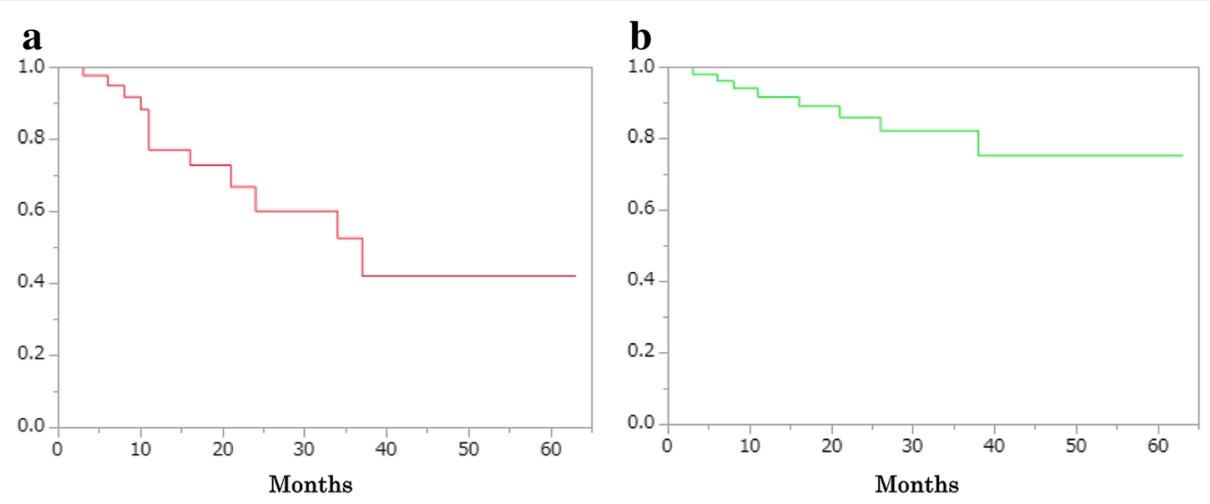

Fig. 1 a Stone recurrence-free survival rate and $\mathbf{b}$ Stone-related event-free survival rate in operation group 

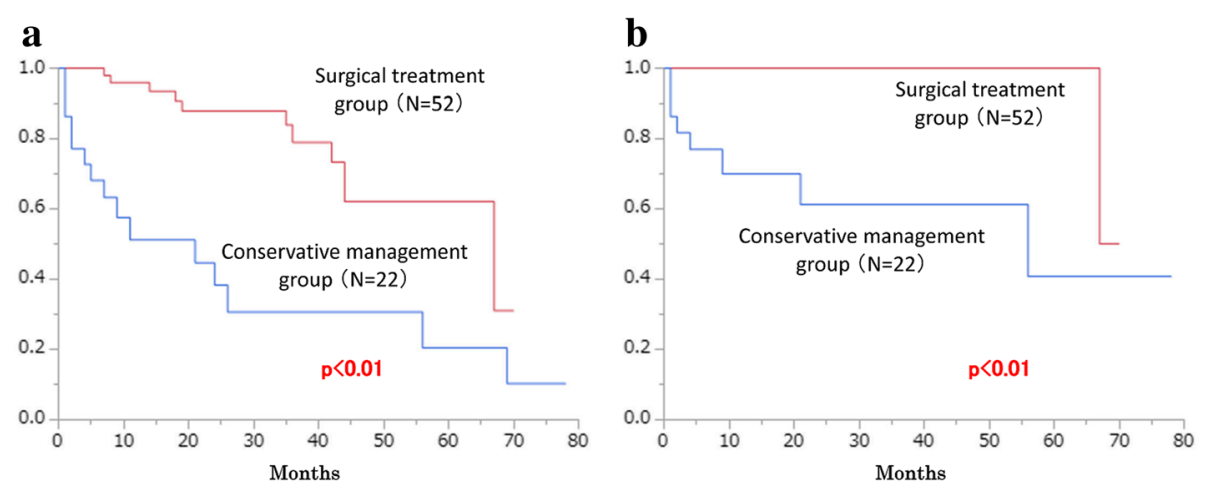

Fig. 2 Comparison of a overall survival rate and $\mathbf{b}$ stone-specific survival rate between operation group and non-operation group

observed in only one patient and no patients expired perioperatively. These results suggest that perioperative complications were acceptable, given the comorbidities in patients with poor PS. In spite of this, these results do not necessarily mean that active stone removal, even for patients in conservative management group, could be performed safely. Patient backgrounds are different between surgical treatment and conservative management groups. We suggest that active stone removal for patients with poor PS could be performed safely as long as perioperative risks are assessed and managed carefully.

Second, results of the survival analyses suggested that patients who had active interventions achieved longer survival in spite of their poor PS. Another reason why physicians choose conservative treatment for patients with poor PS is the assumption that they have poor prognoses because of the multiple comorbidities. Slot et al. reported that the median survival of patients with poor PS resulting from ischaemic stroke was 2.5 years after the stroke [3]. Xie et al. reported that the median survival of patients with dementia was 4.5 years after the onset [2]. Hossain et al. reported that approximately one in five people with spinal cord injury who are wheelchair-dependent die within 2 years of discharge from hospital [10]. Considering the results of these studies, it is questionable whether active stone removal is recommended for patients with poor PS. However, to our knowledge, there are no published studies related to the influence of stone removal on patients with poor PS.

In the present study, the two-year OS rate in the surgical treatment group (88.0\%) was better than that in the conservative management group (38.4\%). Multivariate Cox proportional hazards regression model shows that stone removal is the only independent predictor of SSS. In addition, stone removal is not significant, but is considered a possible favorable predictor of OS. These survival analyses showed that the patients who had active interventions achieved their longer survival in spite of their poor PS. However, these results do not necessarily suggest that active stone removal in patients with poor PS could prevent stone-related death and may improve their prognosis, because patient characteristics are different between two groups. The median age of patients in the conservative management group was 86 years, significantly older than that in the surgical treatment group $(p<0.01)$. Moreover, preoperative pyelonephritis seemed to be more frequently observed in the conservative management group compared with the surgical treatment group $(p=0.09)$. Although we adjusted the patient backgrounds using various factors, such as age, sex,

Table 3 Univariate and multivariate analyses of associations between various parameters and overall survival

\begin{tabular}{|c|c|c|c|c|c|c|}
\hline \multirow[t]{2}{*}{ Variable } & \multicolumn{3}{|c|}{ Univariate analysis } & \multicolumn{3}{|c|}{ Multivariate analysis } \\
\hline & $\overline{\mathrm{HR}}$ & $95 \% \mathrm{Cl}$ & $p$ value & $\overline{\mathrm{HR}}$ & $95 \% \mathrm{Cl}$ & $p$ value \\
\hline Age, year & 1.09 & $1.05-1.15$ & $<0.01$ & 1.08 & $1.02-1.15$ & $<0.01$ \\
\hline Female (vs Male) & 3.10 & $1.17-10.70$ & 0.02 & 1.92 & $0.63-7.41$ & 0.26 \\
\hline Charlson Comorbidity Index & 1.28 & $0.98-1.64$ & 0.06 & 1.36 & $1.10-1.68$ & $<0.01$ \\
\hline Coexisting acute pyelonephritis & 1.53 & $0.58-5.26$ & 0.42 & & & \\
\hline Urinary drainage & 0.94 & $0.38-2.83$ & 0.90 & & & \\
\hline Stone size, $\mathrm{mm}$ & 0.99 & $0.94-1.03$ & 0.52 & & & \\
\hline Multiple stones & 0.89 & $0.41-1.96$ & 0.75 & & & \\
\hline Stone removal & 0.22 & $0.10-0.49$ & $<0.01$ & 0.43 & $0.16-1.09$ & 0.07 \\
\hline
\end{tabular}


Table 4 Univariate and multivariate analyses of associations between various parameters and stone-specific survival

\begin{tabular}{|c|c|c|c|c|c|c|}
\hline \multirow[t]{2}{*}{ Variable } & \multicolumn{3}{|c|}{ Univariate analysis } & \multicolumn{3}{|c|}{ Multivariate analysis } \\
\hline & $\mathrm{HR}$ & $95 \% \mathrm{Cl}$ & $p$ value & $\mathrm{HR}$ & $95 \% \mathrm{Cl}$ & $p$ value \\
\hline Age, year & 1.10 & $1.02-1.22$ & $<0.01$ & 1.01 & $0.91-1.14$ & 0.90 \\
\hline Female (vs Male) & 4.59 & $0.83-85.69$ & 0.08 & 3.80 & $0.39-95.67$ & 0.27 \\
\hline Charlson Comorbidity Index & 1.21 & $0.79-1.78$ & 0.36 & & & \\
\hline Coexisting acute pyelonephritis & 1.99 & $0.36-37.98$ & 0.47 & & & \\
\hline Urinary drainage & 0.43 & $0.11-2.07$ & 0.26 & & & \\
\hline Stone size, mm & 0.97 & $0.86-1.04$ & 0.45 & & & \\
\hline Multiple stones & 1.55 & $0.40-7.39$ & 0.53 & & & \\
\hline Stone removal & 0.05 & $0.00-0.27$ & $<0.01$ & 0.06 & $0.00-0.43$ & $<0.01$ \\
\hline
\end{tabular}

coexisting acute pyelonephritis and CCI, as possible in multivariate Cox proportional hazards regression model, other differences which are not reflected in these factors could have a strong influence on the patients' prognosis. Therefore, randomized controlled trials or a large-scale propensity score matching analysis using even more factors are necessary to make clear whether active stone removal in patients with poor PS could prevent stonerelated death and could improve their prognosis.

Third, active stone removal for patients with poor PS could prevent stone-related events. In the present study, overall SFR in the surgical treatment group was $78.8 \%$. This is acceptable considering the adverse conditions specific to patients with poor PS. However, when new stone formation and regrowth of residual fragments were included in the definition of recurrence, two-year and five-year RFS rates were low at $60.2 \%$ and $42.1 \%$, respectively. This might be because of risks of stone formation specific to patients with poor PS such as hypercalciuria associated with osteoporosis, urinary stasis, urinary tract infection and low fluid intake. In addition, prevention of stone recurrence is also difficult in these patients because of decreased accessibility to medical services and compliance to fluid intake and medication. However, given the fact that their life expectancy is generally short, we believe that it is more important to prevent stone-related symptoms and avoid further interventions for stones, rather than preventing radiographic recurrence. From that point of view, twoyear EFS rate of $86.0 \%$ and five-year EFS rate of $75.4 \%$ were considered to be satisfactory.

To reduce the recurrence rate, the achievement of stone-free status is important. In our study, by the operative treatment method, SFR was $50.0 \%$ in SWL, $87.2 \%$ in URS and $50.0 \%$ in PCNL, respectively. The main cause of the low SFR in PCNL cases was considered to be larger stone size (median $27.5 \mathrm{~mm}$, range 9-53 $\mathrm{mm}$ ) compared with other operations. On the other hand, the reasons for the low SFR in SWL might include the difficulty of spontaneous expulsion of fragments after lithotripsy because of low fluid intake and physical activities in patients with poor PS. Therefore, our results suggest that URS is preferable to SWL in the treatment of patients with poor PS.

There are several limitations to the present study. First, this was a retrospective study undertaken at several centers with a relatively small number of patients. Second, treatment policy was left to the judgment of attending physicians, patients and their families. In addition, this study targeted only hospitalized patients and did not include most patients with asymptomatic calculi.

Despite these limitations in our study, we were able to demonstrate that active stone removal for patients with poor PS could be performed safely and the patients who had active interventions achieved their longer survival with infrequent stone-related events as long as perioperative risks are assessed and managed carefully. To establish the guideline for the optimal management of urolithiasis in these patients, further prospective analysis involving a multicenter approach is required. In addition, an effective method to prevent stone recurrence is essential as it has been a challenge particularly for the patients with poor PS.

\section{Conclusions}

Active stone removal for patients with poor PS could be performed safely and effectively. Compared to conservative management, surgical stone treatment achieved longer OS and SSS.

\section{Additional file}

Additional file 1: Table S1. The demographics and stone

characteristics of patients, de-identified raw data. (XLSX 23 kb)

\section{Abbreviations}

CCl: Charlson Comorbidity Index; ECOG: Eastern Cooperative Oncology Group; EFS: Stone-related event-free survival; NCCT: Non-contrast computed tomography; OS: Overall survival; PCNL: Percutaneous nephrolithotomy; PS: Performance status; RFS: Recurrence-free survival; SFR: Stone-free rate; SSS: Stone-specific survival; SWL: Shock wave lithotripsy; URS: Ureteroscopy 


\section{Acknowledgements}

We would like to thank Prof Shimokawa for assistance in statistical analysis.

\section{Funding}

This study was supported by third research grant from the Japanese Society on Urolithiasis Research. The study protocol has been peer-reviewed by the funding body.

\section{Availability of data and materials}

All data generated or analysed during this study are included in this published article and its supplementary information files. Identifying information has been removed to protect patient identities.

\section{Authors' contributions}

SY and YK designed the study. YH, TI, Al, MH, HK, TW and SN acquired the data. SY performed statistical analyses and drafted the manuscript. SY, YK and $\mathrm{IH}$ interpreted study results and finalized the manuscript. All authors read and approved the final manuscript.

\section{Ethics approval and consent to participate}

This study was approved by the institutional review board of Wakayama Medical University. While written informed consent to participate in this study was not obtained from patients since this study was a retrospective observational study for ordinary medical practice, information about this clinical study was disclosed on the WMUH web page and displayed at the hospital's visitor consultation rooms.

\section{Consent for publication}

Not applicable.

\section{Competing interests}

The authors declare that they have no competing interests.

\section{Publisher's Note}

Springer Nature remains neutral with regard to jurisdictional claims in published maps and institutional affiliations.

\section{Author details}

'Department of Urology, Wakayama Medical University, 811-1 Kimiidera, Wakayama City, Wakayaka 641-0012, Japan. ²Department of Urology, Hashimoto Municipal Hospital, 2-8-1 Ominedai, Hashimoto City, Wakayama 648-0005, Japan. ${ }^{3}$ Department of Urology, Kinan Hospital, 46-70 Shinjyo, Tanabe City, Wakayama 646-8588, Japan. ${ }^{4}$ Department of Urology, Rinku General Medical Center, 2-23 Rinkuouraikita, Izumisano City, Osaka 598-8577, Japan.

Received: 25 May 2017 Accepted: 31 October 2017

Published online: 16 November 2017

\section{References}

1. United Nations World Population Prospects:The 2012 Revision (http://esa.un org/unpd/wpp/)

2. Xie J, Brayne C, Matthews FE. Survival times in people with dementia: analysis from population based cohort study with 14 year follow-up. BMJ (Clinical research ed). 2008;336(7638):258-62

3. Slot KB, Berge E, Dorman P, Lewis S, Dennis M, Sandercock P. Impact of functional status at six months on long term survival in patients with ischaemic stroke: prospective cohort studies. BMJ (Clinical research ed). 2008;336(7640):376-9.

4. Mitchell SL, Teno JM, Kiely DK, Shaffer ML, Jones RN, Prigerson HG, Volicer L, Givens JL, Hamel MB. The clinical course of advanced dementia. N Engl J Med. 2009;361(16):1529-38.

5. Nabbout P, Slobodov G, Culkin DJ. Surgical management of urolithiasis in spinal cord injury patients. Current urology reports. 2014;15(6):408.

6. Guidelines on Urolithiasis.European Association of Urology. (http://uroweb. org/guideline/urolithiasis/)

7. Logarakis NF, Jewett MA, Luymes J, Honey RJ. Variation in clinical outcome following shock wave lithotripsy. J Urol. 2000;163(3):721-5.

8. Labate G, Modi P, Timoney A, Cormio L, Zhang X, Louie M, Grabe M, Rosette On Behalf Of The Croes Pcnl Study Group J. The percutaneous nephrolithotomy global study: classification of complications. Journal of endourology/Endourological Society. 2011:25(8):1275-80.

9. Seitz C, Desai M, Hacker A, Hakenberg OW, Liatsikos E, Nagele U, Tolley D. Incidence, prevention, and management of complications following percutaneous nephrolitholapaxy. Eur Urol. 2012;61(1):146-58.

10. Hossain MS, Rahman MA, Herbert RD, Quadir MM, Bowden JL, Harvey LA. Two-year survival following discharge from hospital after spinal cord injury in Bangladesh. Spinal Cord. 2016;54(2):132-6.

\section{Submit your next manuscript to BioMed Central and we will help you at every step:}

- We accept pre-submission inquiries

- Our selector tool helps you to find the most relevant journal

- We provide round the clock customer support

- Convenient online submission

- Thorough peer review

- Inclusion in PubMed and all major indexing services

- Maximum visibility for your research

Submit your manuscript at www.biomedcentral.com/submit 